\begin{tabular}{|c|l|}
\hline Title & Control of spontaneous emission coupling factor $\beta$ in fiber-coupled microsphere resonators \\
\hline Author(s) & Takashima, Hideaki; Fujiwara, Hideki; Takeuchi, Shigeki; Sasaki, Keiji; Takahashi, Masahide \\
\hline Citation & $\begin{array}{l}\text { A pplied Physics Letters, 92/7), 071115-1-071115-3 } \\
\text { https://doi.org/_0.1063/2884329 }\end{array}$ \\
\hline Issue Date & 2008-02-21 \\
\hline Doc URL & http://hdl.handle.net/2115/32714 \\
\hline Rights & ○ 2008 A merican Institute of Physics. \\
\hline Type & article \\
\hline File Information & A pplPhysLett_92_071115.pdf \\
\hline
\end{tabular}

Instructions for use 


\title{
Control of spontaneous emission coupling factor $\beta$ in fiber-coupled microsphere resonators
}

\author{
Hideaki Takashima, ${ }^{1}$ Hideki Fujiwara, ${ }^{1}$ Shigeki Takeuchi, ${ }^{1, a)}$ Keiji Sasaki, ${ }^{1}$ and \\ Masahide Takahashi ${ }^{2}$ \\ ${ }^{1}$ Research Institute for Electronic Science, Hokkaido University, Sapporo 060-0812, Japan \\ ${ }^{2}$ Institute for Chemical Research, Kyoto University, Uji, Kyoto 611-0011, Japan
}

(Received 12 November 2007; accepted 24 January 2008; published online 21 February 2008)

\begin{abstract}
The spontaneous emission coupling factor $\beta$ is studied for fiber-coupled microspheres with a thin gain layer of phosphorus codoped sol-gel erbium silica-aluminum glass. From the input-output characteristics of the lasing, $\beta=0.039$ is estimated for a sample with a gain layer on the sphere surface. $\beta$ is estimated to increase to 0.19 when an additional silica glass thin layer is overcoated to improve the overlap between the gain layer and the optical mode of the lasing inside the sphere. (C) 2008 American Institute of Physics. [DOI: 10.1063/1.2884329]
\end{abstract}

Microsphere resonators coupled with tapered optical fibers have attracted considerable attention recently as ideal cavity systems with ultrahigh $Q$ factors $\left[Q>10^{8}\right.$ (Ref. 1)] and single spatial mode input-outputs. Pioneering works have been done on coupling between a silica microsphere and a tapered fiber. ${ }^{2,3}$ More recently, micro-optical devices such as microsphere lasers and add-drop filters have been demonstrated. $^{4-9}$ In addition to $Q$ values and coupling efficiency, a crucial parameter for its applications is the spontaneous emission coupling factor $\beta$, defined as the fraction of spontaneous emission coupled into a cavity mode with respect to the spontaneous emission into all modes. ${ }^{10}$ To realize microsphere lasers with a low lasing threshold, it is essential to improve $\beta$. Moreover, $\beta$ is important for the realization of optical quantum information devices, such as single photon sources ${ }^{11}$ and quantum phase gates. ${ }^{12,13}$

The $\beta$ factor has been investigated in sole liquid microdroplets and polymer microspheres (without tapered-fiber coupling). Chang and Campillo measured the stimulated Raman scattering in carbon disulfide microdroplets of $8 \mu \mathrm{m}$ diameter. ${ }^{14}$ They observed an absence of a clear threshold and estimated $\beta$ to be about 0.42 . Kuwata-Gonokami and Takeda demonstrated lasing in a $5 \mu \mathrm{m}$ diameter polystyrene microsphere with a dye doped surface layer. ${ }^{15}$ From the input and output characteristics near the lasing threshold, they estimated $\beta$ to be 0.3 . However, these studies were for sole microspheres using free-space laser pumping and $\beta$ factors for fiber-coupled microspheres have not been fully investigated.

In this letter, we report the estimation of $\beta$ factors for fiber-coupled microspheres. We also study the change of $\beta$ factor according to the location of the gain layer inside the sphere. For this study, the thickness of the gain layer has to be sufficiently smaller than the distribution of the optical mode in the sphere. For this purpose, we used a method to fabricate a fiber-coupled microsphere laser with a submicron thickness gain layer. ${ }^{9}$ By overcoating an undoped sol-gel silica glass thin layer to improve the overlap between the gain layer and the optical mode in the sphere, $\beta$ of a sample with a gain layer on the sphere surface was increased from 0.039 to 0.19 .

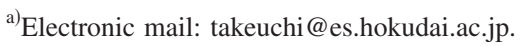

Overcoating of an undoped sol-gel silica glass on a precoated gain layer produces optimal coupling between the gain layer and the optical mode in a microsphere. Figure 1 shows the calculated distribution of the thin gain layer and the optical mode in a $40 \mu \mathrm{m}$ diameter microsphere at $1535.7 \mathrm{~nm}$ wavelength, corresponding to the emission wavelength of Er. The calculation of the optical mode was based on the Lorenz-Mie theory. ${ }^{16}$ The optical mode is confined in the region about $600 \mathrm{~nm}$ inside from the surface with full width at half maximum of about $1000 \mathrm{~nm}$. To realize a large $\beta$, it is essential to locate the gain layer at the peak of the optical mode. Considering the thickness of the gain layer (200 nm), optimal coupling can be achieved by overcoating a sol-gel silica glass of about $500 \mathrm{~nm}$ thickness on the precoated gain layer.

Samples were prepared as follows. A microsphere surface coated with $\mathrm{Er}^{3+}$-doped $\mathrm{P}_{2} \mathrm{O}_{5}-\mathrm{Al}_{2} \mathrm{O}_{3}-\mathrm{SiO}_{2}$ thin films (sample A) was fabricated using a previously described method. ${ }^{9}$ The coating sol for the gain layer was prepared by stirring ethanol (EtOH), dimethylformamide, tetraethoxysilane (TEOS), hydrochloric acid $(\mathrm{HCl})$, aluminum chloride $\left(\mathrm{AlCl}_{3}\right)$, erbium chloride hexahydrate $\left(\mathrm{ErCl}_{3} \cdot 6 \mathrm{H}_{2} \mathrm{O}\right)$, and triethylphosphate $\left[\left(\mathrm{C}_{2} \mathrm{H}_{5} \mathrm{O}\right)_{3} \mathrm{PO}\right]$. Silica microspheres with stems were formed by melting the edge of a tapered fiber tip using a $\mathrm{CO}_{2}$ laser. The microsphere was immersed in the coating sol for $30 \mathrm{~s}$. After withdrawing the microsphere at a

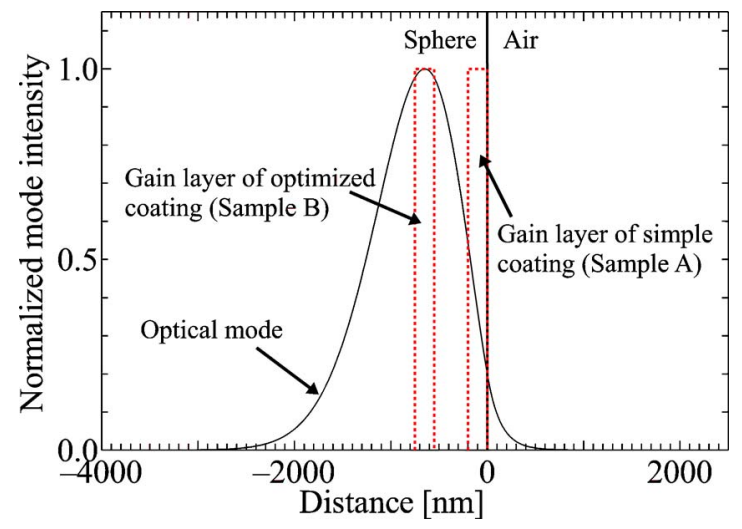

FIG. 1. (Color online) Typical gain layer distribution with $200 \mathrm{~nm}$ thickness (dotted line) and angle-averaged radial intensity of the optical mode (solid line) in a $40 \mu \mathrm{m}$ diameter microsphere at $\lambda=1535.7 \mathrm{~nm}$. 
speed of $3 \mathrm{~cm} / \mathrm{min}$ from the solution, the microsphere was annealed in an electrical furnace at $900{ }^{\circ} \mathrm{C}$ for $10 \mathrm{~min}$. Then, the microsphere was irradiated with a $\mathrm{CO}_{2}$ laser (about $3.5 \mathrm{~W}$ ) for a few tens of seconds to anneal out microcracking in the sol-gel surface. ${ }^{5}$ The molar composition of the fabricated films was $\mathrm{SiO}_{2}: \mathrm{Al}_{2} \mathrm{O}_{3}: \mathrm{P}_{2} \mathrm{O}_{5}: \mathrm{Er}_{2} \mathrm{O}_{3}$ $=1: 0.12: 0.05: 0.01$, corresponding to a concentration of $\mathrm{Er}$ ions of $10^{4} \mathrm{ppm}$. In order to estimate the thickness of the films on the microsphere, the thicknesses of the thin films fabricated on the surface oxidized silicon substrate were analyzed using an ellipsometer. The thickness of the thin film was estimated to be $200 \mathrm{~nm}$. The diameter of the microsphere was $40 \mu \mathrm{m}$.

A microsphere with an undoped sol-gel silica glass layer (sample B) was fabricated as follows. For the coating sol of this glass, a mixture of $\mathrm{EtOH}, \mathrm{HCl}$, TEOS, and $\mathrm{H}_{2} \mathrm{O}$ was stirred for about $19 \mathrm{~h}$. The molar ratio of the undoped sol-gel silica layer was $\mathrm{EtOH}: \mathrm{HCl}: \mathrm{TEOS}: \mathrm{H}_{2} \mathrm{O}=1: 0.04: 0.15: 1.54$. After the same procedure for sample $\mathrm{A}$, the microsphere with a gain layer on the surface without $\mathrm{CO}_{2}$ laser annealing was dipped into the coating sol and then annealed at $900{ }^{\circ} \mathrm{C}$ for $10 \mathrm{~min}$. Since the thickness of the glass layer formed by one coating process was estimated to be $150 \mathrm{~nm}$, we repeated this process three times in order to achieve a thickness of about $450 \mathrm{~nm}$. After the overcoating procedure, the microsphere was irradiated with a $\mathrm{CO}_{2}$ laser for a few seconds. The diameter of the microsphere was $45 \mu \mathrm{m}$, almost the same as that of sample A.

Tapered fibers were fabricated by heating a single-mode fiber (Thorlabs, 1060XP) with a ceramic heater and stretching the end of the fiber. ${ }^{17,18}$ The transmittance of the fiber was about $90 \%$ (when fabricated) and flat for the wavelength region from 1500 to $1620 \mathrm{~nm}$, indicating single-mode operation at $1550 \mathrm{~nm}^{18}$

A single-mode laser diode operating at $975 \mathrm{~nm}$ (Thorlabs, PL980P200) with single-mode optical fiber output was used for pumping. ${ }^{9}$ The intensity of the pump light was controlled using a variable optical attenuator. A prepared microsphere sample was gently touched to the tapered fiber under microscope observation using a two-dimensional piezomanipulator. During the experiments, the microspheres were in contact with the tapered fiber inside a plastic box filled with dry air. ${ }^{17}$ Unabsorbed pump light $(975 \mathrm{~nm})$ was removed from the laser emission light (about $1550 \mathrm{~nm}$ ) using two $980 / 1550 \mathrm{~nm}$ wave division multiplexing couplers. Then, the laser emission light was analyzed using a spectrometer (Oriel, MS257) equipped with an InGaAs multichannel detector head (Hamamatsu, C8062-01).

Figure 2(a) shows the single-mode lasing spectrum of sample A (microsphere with a surface gain layer). The estimated pump power in the tapered fiber was $6.7 \mathrm{~mW}$. Figure 2(b) shows the lasing peak versus the estimated pump power on logarithmic scales. The upper and lower dotted lines are fits to the regions of spontaneous emission and stimulated emission, respectively. $\beta$ can be estimated from the ratio of the output intensity below and above the lasing threshold. ${ }^{19}$ Since the ratio of the output intensity at the lasing threshold $(1.6 \mathrm{~mW})$ is $25.7, \beta$ as the inverse of this ratio was estimated to be 0.039 .

Figure 3(a) shows the single-mode lasing spectrum of sample B (microsphere with optimized coupling). The estimated pump power in the tapered fiber was $3.3 \mathrm{~mW}$. Figure 3 (b) shows the lasing peak versus the estimated pump power.
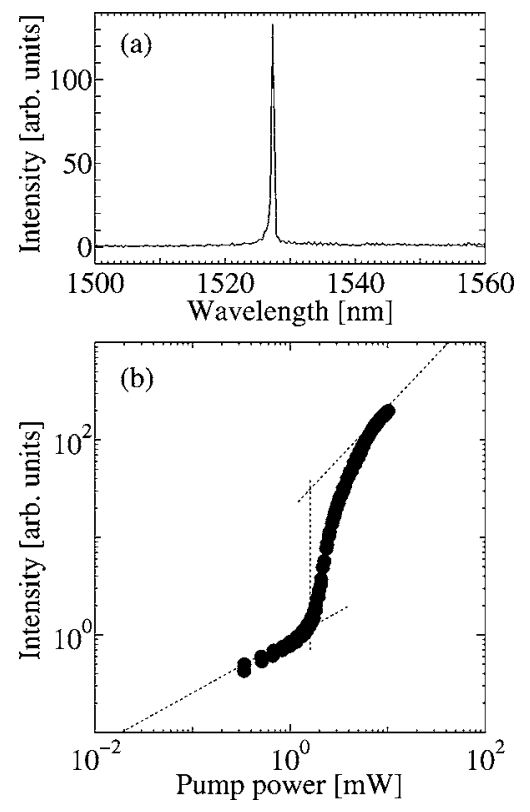

FIG. 2. (a) Single-mode lasing spectrum of sample A. (b) Lasing output intensity as a function of input pump power. The intensities of the upper and lower dotted lines at the rising edge (pump power $=1.6 \mathrm{~mW}$ ) are 30.8 and 1.2 , respectively. Thus, $\beta$ was estimated to be $0.039=1.2 / 30.8$.

The change of the slope around the threshold is more insignificant than for sample A. The ratio of the output intensity below and above the lasing threshold $(0.78 \mathrm{~mW})$ decreases to 5.2. From this ratio, $\beta$ for sample $\mathrm{B}$ was estimated to be 0.19 . We believe that the increase of $\beta$ factor from 0.039 to 0.19 is due to better coupling between the gain layer and the optical mode inside the sphere.

In conclusion, we have reported the estimation of $\beta$ factors for fiber-coupled microspheres and studied the change of $\beta$ factor according to the location of the gain layer inside the sphere. We used a fiber-coupled microsphere laser with a submicron thickness gain layer. ${ }^{9}$ By overcoating an undoped
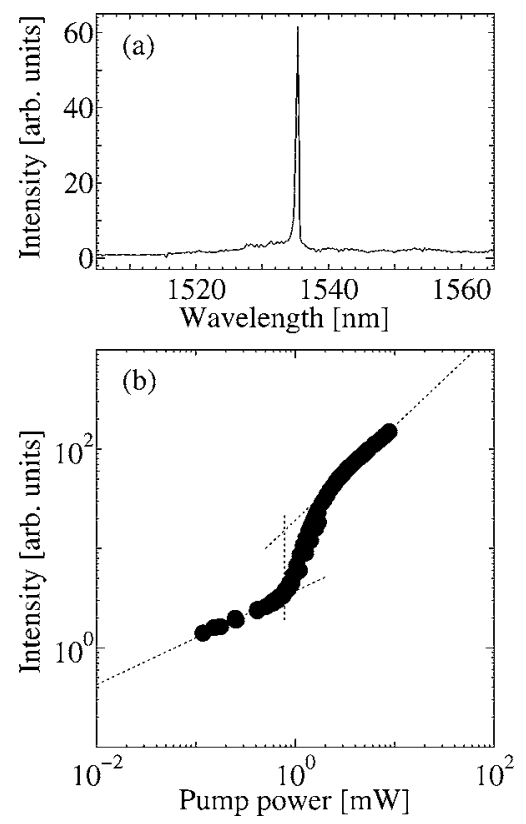

FIG. 3. (a) Single-mode lasing spectrum of sample B. (b) Lasing output intensity as a function of input pump power. The intensities of the upper and lower dotted lines at the rising edge (pump power $=0.78 \mathrm{~mW}$ ) are 17.1 and 3.3, respectively. Thus, $\beta$ was estimated to be $0.19=3.3 / 17.1$. 
sol-gel silica glass thin layer to improve the overlap between the gain layer and the optical mode in the sphere, $\beta$ was increased from 0.039 to 0.19 for a sample with a gain layer on the sphere surface.

The authors would like to thank Koji Uemura for technical assistance. The current work was partly supported by the program " $R \& D$ support scheme for funding selected IT proposals" of the Ministry of Public Management, Home Affairs, Posts and Telecommunications, a Grant-in-Aid from the Japan Society for the Promotion of Science, the 21st century COE program, CREST-project, Japan Science and Technology Agency, and Special Coordination Funds for Promoting Science and Technology.

${ }^{1}$ M. L. Gorodetsky, A. A. Savchenkov, and V. S. Ilchenko, Opt. Lett. 21, 453 (1996).

${ }^{2}$ J. C. Knight, G. Cheung, F. Jacques, and T. A. Birks, Opt. Lett. 22, 1129 (1997).

${ }^{3}$ M. Cai and K. Vahala, Opt. Lett. 25, 260 (2000).

${ }^{4}$ M. Cai, O. Painter, and K. J. Vahala, Opt. Lett. 25, 1430 (2000).

${ }^{5}$ L. Yang and K. J. Vahala, Opt. Lett. 28, 592 (2003).

${ }^{6}$ P. V. Hoi, C. T. T. Ha, and H. Q. Hung, Appl. Phys. Lett. 87, 161110
(2005).

${ }^{7}$ M. Cai, G. Hunziker, and K. J. Vahala, IEEE Photonics Technol. Lett. 6, 686 (1999).

${ }^{8}$ J. Kalkman, A. Polman, T. J. Kippenberg, K. J. Vahala, and M. L. Brongersma, Nucl. Instrum. Methods Phys. Res. B 242, 182 (2006).

${ }^{9}$ H. Takashima, H. Fujiwara, S. Takeuchi, K. Sasaki, and M. Takahashi, Appl. Phys. Lett. 90, 101103 (2007).

${ }^{10}$ H. Yokoyama and S. D. Brorson, J. Appl. Phys. 66, 4801 (1989).

${ }^{11}$ M. Pelton, C. Santori, J. Vučković, B. Zhang, G. S. Solomon, J. Plant, and Y. Yamamoto, Phys. Rev. Lett. 89, 233602 (2002).

${ }^{12}$ H. F. Hofmann, K. Kojima, S. Takeuchi, and K. Sasaki, J. Opt. B: Quantum Semiclassical Opt. 5, 218 (2003).

${ }^{13}$ H. Oka, H. F. Hofmann, S. Takeuchi, and K. Sasaki, Jpn. J. Appl. Phys., Part 1 43, 7495 (2004)

${ }^{14}$ Optical Processes in Microcavities, edited by R. K. Chang and A. J. Campillo (World Scientific, Singapore, 1996).

${ }^{15}$ M. Kuwata-Gonokami and K. Takeda, Opt. Mater. (Amsterdam, Neth.) 9, 12 (1998).

${ }^{16}$ Light Scattering by Particles: Computational Methods, edited by P. W. Barber and S. C. Hill (World Scientific, Singapore, 1990).

${ }^{17}$ A. Chiba, H. Fujiwara, J. Hotta, S. Takeuchi, and K. Sasaki, Appl. Phys. Lett. 86, 261106 (2005)

${ }^{18}$ H. Konishi, H. Fujiwara, S. Takeuchi, and K. Sasaki, Appl. Phys. Lett. 89, 121107 (2006).

${ }^{19}$ R. J. Horowicz, H. Heitmann, Y. Kadota, and Y. Yamamoto, Appl. Phys. Lett. 61, 393 (1992). 